\title{
IN VITRO HYDROXYLATION OF STEROIDS BY WHOLE ADRENAL HOMOGENATES OF BEEF, NORMAL MAN, AND PATIENTS WITH THE ADRENOGENITAL SYNDROME ${ }^{1}$
}

\author{
By ALFRED M. BONGIOVANNI WITH THE TECHNICAL ASSISTANCE OF \\ WILFRIED EDER \\ (From the Endocrine Division, Children's Hospital of Philadelphia, and Department of \\ Pediatrics, University of Pennsylvania, Philadelphia, Pa.)
}

(Submitted for publication May 5, 1958; accepted June 12, 1958)

The partial or complete failure of the adrenal cortex to realize the biosynthesis of $\Delta^{4}$-pregnene$11 \beta, 17 \alpha, 21$-triol-3, 20-dione (compound F) represents the basic defect in patients with the adrenogenital syndrome due to congential adrenocortical hyperplasia (1-5). The most common form of this disorder (with normal blood pressure and without undue salt loss) has been indirectly attributed to a deficiency of adrenal " 21 hydroxylase" as witnessed by the large quantities of urinary $3 \alpha, 17 \alpha, 20 \alpha$-pregnanetriol (hereafter designated "pregnanetriol") and the relatively small amounts of steroids with an oxygen function at carbon $21(4,6,7)$.

The availability of small amounts of human adrenal tissue has made it possible to examine the hydroxylation of some steroid substrates by normal and diseased glands in vitro. Due to the difficulties of obtaining any but limited fresh human material, fresh beef and postmortem human adrenals have also been employed for purposes of comparison. The experiments to be described were designed to measure the introduction of hydroxyl groups into steroid nucleus at positions 11 and 21 only. The appropriate adrenal enzymatic systems will be termed "11-hydroxylase" and " 21 hydroxylase" after Dorfman (8), although it may be that each comprises more than a single component (9).

\section{MATERIALS AND METHODS}

Two normal "fresh" human adrenal glands were obtained from children during renal surgery. Several beef adrenals were collected from a slaughter house within an hour after death. These were frozen immedi-

1 This work was supported by a grant (P-5) from the American Cancer Society and a grant [A-619(C3)] from the Division of Research Grants and Fellowships of the National Institutes of Health, United States Public Health Service. ately after removal and stored at $-10^{\circ}$ C. until used. A single normal gland from a child was taken, within 3 hours after death due to complications during cardiac surgery. In addition, "normal" adult glands were collected at the autopsy table within 24 hours after death. All glands were kept frozen and employed within two weeks of collection.

Adrenal glands from two children with the adrenogenital syndrome were available. One gland $(\mathrm{Bz})$ was removed from a two and one-half year old female pseudohermaphrodite who had never been treated with steroids; the excretion of $17-$ ketosteroids was 13.1 to $15.0 \mathrm{mg}$. per day and the pregnanetriol, 15.0 to $18.0 \mathrm{mg}$. per day (normal in this laboratory: less than $1.0 \mathrm{mg}$. of either at this age). The left gland (weight, $14.0 \mathrm{Gm}$.) was excised during an exploratory operation and frozen immediately. The diseased glands (Sh), kindly sent us by Doctor Thomas Sheppard of Washington University, Seattle, were removed 18 hours after death from an infant, aged 18 months, and stored in the frozen state. The latter patient was on small, inadequate doses of cortisone at the time of death; the urinary 17-ketosteroids were $2.2 \mathrm{mg}$. per day and the pregnanetriol was not measured. This infant had the "salt-losing" form of the disease; in our experience, this variety has always been characterized by greatly elevated quantities of urinary pregnanetriol (2).

The preparation of the tissue, conditions of incubation, addition of adenosine triphosphate (ATP), diphosphopyridine nucleotide (DPN) and fumarate, dialysis and extraction were according to Hayano and Dorfman (10, 11 ), except that the whole gland was used and methylene chloride was substituted for chloroform. These workers reported satisfactory activity with frozen glands upon the addition of ATP and DPN. A tissue to steroid ratio of approximately 1,000 to 1 was generally employed, although this was occasionally exceeded with postmortem material as detailed in the data. In several instances, adrenal homogenates were examined without additional substrate or incubation in order to measure the baseline quantities of some steroids.

Two steroids were added to the incubates, separately, as substrates: $\Delta^{4}$-pregnene-17 $\alpha$-ol-3,20-dione (17 hydroxyprogesterone, designated " $17-H P$ "), and $\Delta^{4}$-pregnene-17 $\alpha$, 21-diol-3,20-dione (hereafter designated "11-desoxycortisol"). The total recovery, after the addition of 17-HP, was measured in aliquots of the extracts in methanol, 
by the ultraviolet absorption at 225, 240 and $255 \mathrm{~m} \mu$ employing a correction factor according to Allen (12). When 11-desoxycortisol was the substrate, suitable aliquots of the entire extract were examined by the PorterSilber reagent (13) in order to determine the total recovery. This amount represents the basis for the calculation of the percentage conversion.

The extracts of the incubates were applied to paper chromatograms. The products sought, depending upon the substrate employed, were cortisol, 11-desoxycortisol and $\Delta^{4}$-pregnene-11 $\beta, 17 \alpha$-diol-3,20-dione (21-desoxycortisol) ; and for baseline studies, in addition to the preceding compounds, $17 \alpha$-hydroxyprogesterone. Several paper chromatograms were employed for each extract so that the quantity of steroid applied did not exceed $0.5 \mathrm{mg}$. per sheet, 6 inches in width. Suitable standards were always included along a narrow edge $(0.5$ inch) and the descending technique was employed as described elsewhere (14). Two solvent systems were used: for the measurement of cortisol and 11-desoxycortisol, system E6, containing $335 \mathrm{ml}$. iso-octane, $165 \mathrm{ml}$. toluene, 175 $\mathrm{ml}$. tert.-butanol, $175 \mathrm{ml}$. methanol, and $100 \mathrm{ml}$. water; and for 17-hydroxyprogesterone and 21-desoxycortisol, system E4 (14), containing $500 \mathrm{ml}$. benzene, $250 \mathrm{ml}$. methanol, and $250 \mathrm{ml}$. water. All solvents were redistilled.

The standard compounds and products of incubation resolved by chromatography were located by scanning with ultraviolet light, the Zimmermann reaction as modified in this laboratory (15), triphenyltetrazolium hydrochloride and 4 per cent phosphomolybdic acid in methanol. Further details of this aspect appear elsewhere (16). The portions of the paper containing the extracts, where steroid was detected along a carefully measured narrow strip, were eluted three times with methanol. The quantities of cortisol and 11-desoxycortisol were measured by the Porter-Silber reaction (17) on small aliquots; the 21-methyl steroids were quantitated by the Gornall reaction (18) at the wave length of maximal absorption. Application of pure standards of each compound to paper chromatograms, resolution and elution, as described, yielded recoveries of 68 to 95 per cent.

TABLE I

Steroid content of adrenal glands in micrograms per gram tissue

\begin{tabular}{|c|c|c|c|c|}
\hline Source & Condition & $\mathrm{F}^{*}$ & St & 17-HP \\
\hline $\begin{array}{l}\text { Beef } \\
\text { Human }\end{array}$ & $\begin{array}{l}\mathrm{N} \& \text { fresh frozen } \\
\mathrm{N} \text { fresh frozen } \\
\text { (child) }\end{array}$ & $\begin{array}{l}8.9 \\
4.1\end{array}$ & $\begin{array}{r}11.6 \\
5.6\end{array}$ & $\begin{array}{l}\mathbf{0} \\
\mathbf{0}\end{array}$ \\
\hline Human & $\mathrm{N} \underset{\text { (adult) }}{\text { postmortem }}$ & 6.0 & 5.1 & 4.5 \\
\hline $\begin{array}{l}\mathrm{Bz} \\
\mathrm{Sh}\end{array}$ & $\begin{array}{l}\text { ADH\| fresh frozen } \\
\text { ADH postmortem }\end{array}$ & $\begin{array}{l}0 \\
0\end{array}$ & $\begin{array}{l}0 \\
0\end{array}$ & $\begin{array}{l}118.5 \pi \\
155.5 \pi\end{array}$ \\
\hline
\end{tabular}

* F, 17-hydroxycorticosterone.

† S, 17-hydroxy, 11-desoxycorticosterone.

$\ddagger$ 17-HP, 17-hydroxyprogesterone.

$\$ \mathrm{~N}$, normal.

ADH, congenital adrenal hyperplasia.

If Identified (see text).
The identity of the products depended upon various criteria. The mobility on the chromatograms, the reactions with the reagents applied, and the sulfuric acid chromogens conformed in all instances to the designated compounds. In order to obtain the sulfuric acid chromogens, the eluates from paper were further purified on a small column of silica as described previously (16). In a few specified instances, enough steroid was obtained singly or in pairs of experiments for identification by infrared spectroscopy.

Homogenates of freshly frozen beef adrenal were included in all groups of experiments as an internal control. Several preliminary experiments with beef adrenal (not reported here) were conducted in order to standardize the conditions for this study.

\section{RESULTS}

\section{Steroids in adrenal gland}

The quantity of cortisol, 11-desoxycortisol, and 17-hydroxyprogesterone detected following the chromatography of extracts of unincubated adrenal glands is shown in Table I. The mobilities and staining reactions of cortisol and 11-desoxycortisol, in system E6, as well as the sulfuric acid chromogens, were identical with the designated steroids. There were insufficient amounts for further identification. No alpha-ketolic compounds whatsoever were detected in the two glands from patients with the adrenogenital syndrome. However, relatively large quantities of 17 -hydroxyprogesterone were found in the adrenals from the patients with this disorder, whereas virtually none was present in beef or normal human material. The quantity of tissue extracted from the diseased organs was $2.5 \mathrm{Gm}$. (Bz) and $2.0 \mathrm{Gm}$. (Sh). In system E4, the mobility of the only detectable compound was identical with pure 17-hydroxyprogesterone : $32.5 \mathrm{~cm}$. in seven hours at $23^{\circ} \mathrm{C}$. It did not react with triphenyltetrazolium (TPZ) and gave a blue color with alkaline ethanolic $m$-dinitrobenzene on paper. Following elution and examination of the sulfuric acid chromogens, there was maximal absorbance at wave length 290 and $430 \mathrm{~m} \mu$, similar to the pure standard. The eluates from both glands were combined and oxidized with 2 per cent chromium trioxide in 90 per cent glacial acetic acid. The product revealed a single maximal absorbance in sulfuric acid at $295 \mathrm{~m} \mu$, as for $\Delta^{4}$-androstene-3,17-dione; by infrared spectroscopy, the product was identical with the latter. In spite of diligent efforts to discover the presence 
TABLE II

Conversion of 17-hydroxyprogesterone to compound $F$ and substance $S$ (per cent)

\begin{tabular}{|c|c|c|c|c|c|c|c|}
\hline Source & Condition & Tissue & 17-HP* & $\begin{array}{l}\text { Total } \\
\text { recovery }\end{array}$ & $\mathrm{Ft}$ & Sł & $\begin{array}{c}\text { Total } \\
\text { conversion }\end{array}$ \\
\hline $\begin{array}{l}\text { Beef } \\
\text { Human } \\
\text { Human } \\
\text { Human } \\
\text { Human } \\
\text { Human } \\
\text { Human } \\
\text { Bz } \\
\text { Sh }\end{array}$ & $\begin{array}{ll}\text { FF§ } & \\
\text { FF } & \text { (8 yrs.) } \\
\text { FF } & \text { (6 yrs.) } \\
\text { PM** } & \text { (3 yrs.) } \\
\text { PM } & \text { (adult) } \\
\text { PM } & \text { (adult) } \\
\text { PM } & \text { (adult) } \\
\text { FF†† } & \text { ADH } \\
\text { PM } & \text { ADH }\end{array}$ & $\begin{array}{r}G m . \\
10.0 \\
3.0 \\
4.0 \\
2.5 \\
3.0 \\
2.5 \\
6.0 \\
4.5 \\
2.0\end{array}$ & $\begin{array}{l}m g . \\
9.5 \\
3.0 \\
4.0 \\
2.0 \\
2.5 \\
2.0 \\
4.5 \\
4.5 \\
1.6\end{array}$ & $\begin{array}{r}80.0 \\
91.5 \\
76.5 \\
83.0 \\
85.0 \\
86.0 \\
86.0 \\
100.0 \\
91.0\end{array}$ & $\begin{array}{l}36.0 \| \\
8.5 \\
7.5 \\
4.3 \\
2.4 \\
2.7 \\
2.3 \\
0 \\
0\end{array}$ & $\begin{array}{l}39.6 \| \\
28.2(\mathrm{a}) \Phi \\
22.3(\mathrm{a}) \\
6.0 \\
8.6 \\
13.3(\mathrm{~b}) \rrbracket \\
10.3(\mathrm{~b}) \\
0 \\
0\end{array}$ & $\begin{array}{c}84.6 \\
36.7 \\
34.5 \\
10.3 \\
10.0 \\
16.8 \\
16.9 \\
0 \\
0\end{array}$ \\
\hline
\end{tabular}

* 17-HP, 17-hydroxyprogesterone (substrate).

+ F, 17-hydroxycorticosterone.

¥ S, 17-hydroxy, 11-desoxycorticosterone.

$\S \mathrm{FF}$, fresh frozen.

of 21-desoxycortisol, none was found in any of the adrenals; as little as 5 micrograms of this steroid was detectable by the methods employed, when the pure standard was chromatographed on paper. Elution of the entire paper in the zone corresponding to 21-desoxycortisol yielded no compounds reacting to the Gornall (18) reagent.

\section{7-Hydroxyprogesterone as substrate}

The results of those experiments in which 17 hydroxyprogesterone was added to the incubates are shown in Table II. The results are expressed - as the percentage conversion of the substrate (based on the total amount of steroid recovered) to 11-desoxycortisol or cortisol. In all instances, including postmortem material, a significant degree of 21-hydroxylation was achieved except with the two adrenals from patients with the
$\|$ Identified (infrared).

T a, b, identified in pairs (infrared).

** PM, postmortem.

†† ADH, congenital adrenal hyperplasia.

adrenogenital syndrome. There was usually a greater amount of 11-desoxycortisol than cortisol present, and although this might suggest that the 11-hydroxylating system deteriorated during the experiment, this is not borne out by the observations (Table III) when 11-desoxycortisol was employed as the substrate with the same tissues. It is interesting that 21-hydroxylation and 11hydroxylation were achieved with the postmortem material, although the conversion was less than with the freshly frozen tissue. In some instances, as indicated, the identity of the acetylated products was confirmed by infrared spectroscopy. In all other cases, the criteria for identification outlined above, including the sulfuric acid chromogens, were consistent with the indicated product. Again, despite most careful scrutiny of the paper chromatograms, no 21-desoxycortisol was found in any experiment.

TABLE III

Conversion of substance $S$ to compound $F$ (per cent)

\begin{tabular}{|c|c|c|c|c|c|}
\hline Source & Condition & Tissue & $\mathrm{S}^{*}$ & $\begin{array}{l}\text { Total } \\
\text { recovery }\end{array}$ & $\mathrm{Ft}$ \\
\hline $\begin{array}{l}\text { Human } \\
\text { Beef } \\
\text { Beef } \\
\text { Human } \\
\text { Bz } \\
\text { Sh }\end{array}$ & $\begin{array}{l}\text { FF† (7 yrs.) } \\
\text { FF } \\
\text { FF } \\
\text { PM\| (adult) } \\
\text { FF ADH } \\
\text { PM ADH }\end{array}$ & $\begin{array}{l}G m . \\
2.0 \\
2.5 \\
2.5 \\
3.0 \\
2.5 \\
2.0\end{array}$ & $\begin{array}{l}m g . \\
2.0 \\
2.0 \\
2.0 \\
2.5 \\
2.0 \\
1.6\end{array}$ & $\begin{array}{l}90.0 \\
96.0 \\
93.0 \\
90.0 \\
68.0 \\
95.0\end{array}$ & $\begin{array}{l}49.5 \\
69.0 \\
78.5 \S \\
17.8 \\
77.0(\mathrm{a})^{* *} \\
24.6(\mathrm{a})^{* *}\end{array}$ \\
\hline
\end{tabular}

* S, 17-hydroxy, 11-desoxycorticosterone (substrate).

$\dagger \mathrm{F}, 17$-hydroxycorticosterone.

$\ddagger$ FF, fresh frozen.

$\S$ Identified (infrared).

PM, postmortem.

II ADH, congenital adrenal hyperplasia.

** Identified, paired (infrared). 


\section{1-Desoxycortisol as substrate}

The measurement of 11-hydroxylating activity, employing 11-desoxycortisol as the substrate, is shown in Table III. The yield of cortisol was considerably greater than when the earlier precursor, 17-hydroxyprogesterone (Table II), was employed. The diseased adrenals were capable of converting 11-desoxycortisol to cortisol with even greater facility than comparable normal glands. However, the conditions of the experiment do not allow quantitative assay of the enzymatic systems in the various trials. The ability of one diseased gland (Sh, removed after death) to accomplish this conversion suggests respectable preservation of at least the 11-hydroxylating system, lending credence to the belief that the failure of conversion when 17-hydroxyprogesterone was used as a substrate (Table II) was not due to complete deterioration.

\section{DISCUSSION}

The experiments of Hechter and Pincus (19) by perfusion of beef adrenal glands with various precursors, and of Hayano and Dorfman $(10,11)$ with adrenal homogenates in vitro, gave impetus to the studies described herein. Perusal of these contributions, previous work from this laboratory, and that of many others cited elsewhere (1-5) had suggested that in the nonhypertensive form of the adrenogenital syndrome due to congenital adrenal hyperplasia, the inability to hydroxylate the steroid nucleus at carbon 21 (deficiency of 21-hydroxylase) represented the basis of this disorder. This study gives direct evidence of a deficiency of 21-hydroxylation: Two diseased adrenal glands were unable to convert 17-hydroxyprogesterone into either 11-desoxycortisol or cortisol; a large quantity of 17-hydroxyprogesterone was initially present in each of these glands, and there were no alpha-ketolic steroids as in normal glands. The amounts of cortisol present in normal glands is slightly greater than that reported by others, but considerably more 11-desoxycortisol was found than indicated by Neher (20).

The relatively limited data have been restricted by the difficulties in obtaining fresh human adrenal tissue. It is virtually impossible to obtain tissue from patients with the adrenogenital syndrome since the treatment of this disorder has become primarily medical (21), and indeed the relative adrenocortical insufficiency renders surgery hazardous. One abnormal gland studied $(\mathrm{Bz})$ was obtained under ideal circumstances; the second (Sh) is perhaps less acceptable since it was removed after death from an infant who had received small doses of cortisone. However, certain observations validate the results with the latter. The adrenal content of 17-hydroxyprogesterone of Sh was high, indicating persistent activity in this gland up to the point of 17-HP in the biosynthetic pathway. Other human postmortem tissue obtained after a similar interval of time demonstrated detectable activity with respect to both 21- and 11-hydroxylation, although this was less than with fresh material; significant 11-hydroxylation by this gland was demonstrable, although it may have been less than that obtainable under ideal conditions.

The hydroxylation of various steroid precursors by adrenal tissue in vitro has been described by many others, most of whom employed material from beef $(9,10,22-34)$. The reports have variously indicated the presence of 11-, 17- and 21hydroxylating systems, as well as hydroxylation at other positions. It has been shown that the "11hydroxylase" may be concentrated in the residue and 17- and 21-hydroxylases in the supernatant $(9,27)$ after centrifugation. The limited amounts of tissue available precluded such fractionation here. Lombardo, Roitman and Hudson (30) have studied "normal" human adrenals and Berliner, Berliner and Dougherty (31), the glands from patients with Cushing's syndrome due to adrenal hyperplasia and adrenal carcinoma. The presence of 11-, 17- and 21-hydroxylating enzymes has been detected in these glands from the human subject.

The greater yield of 11-desoxycortisol and cortisol from 17-hydroxyprogesterone noted in these studies, as well as the failure of the diseased adrenal to convert 17-hydroxyprogesterone into detectable quantities of 21-desoxycortisol, is notable. In addition, the more effective transformation of 11-desoxycortisol into cortisol suggests that the human adrenal performs 11-hydroxylation with greater facility after 21-hydroxylation. Hayano and Dorfman have indicated virtually complete transformation of 17-hydroxyprogesterone into cortisol with beef adrenals (11); they did not indicate the presence of 11-desoxycortisol in the 
course of their study. In another report, these workers (10) achieved a 95 per cent transformation of 11-desoxycortisol to cortisol by beef adrenals, an order of conversion greater than reported here. Brownie, Grant and Davidson (32) have shown that progesterone inhibits 11-hydroxylation, and that this may be overcome by the use of succinate; in view of the possibility that the substrates employed in our studies might similarly interfere with 11-hydroxylation, succinate has been added without improvement in the yield. With human adrenal tissue, Lombardo and coworkers (30) have achieved a 50 per cent conversion of 17-hydroxyprogesterone to cortisol; in these experiments, the tissue was used immediately without prior freezing. On the other hand, Berliner and associates (31) presented observations analogous to ours: In one experiment with human tissue there was a greater conversion of progesterone to desoxycorticosterone than to corticosterone and no 11-hydroxyprogesterone was detected. Thus, these workers also observed 21hydroxylated as well as 11-, 21-dihydroxylated products. The prior occurrence of 21-hydroxylation is in accord with the conclusions of Hechter and Pincus (19). The large quantities of 11-hydroxylated 17-ketosteroids reported in the urine of affected individuals (4) remains unexplained by these studies; possibly alternative types of 11hydroxylation occur, since 21-hydroxylation is defective in the disorder. The preponderance of 11-desoxycortisol in our incubates with 17-hydroxyprogesterone, which is unlike other work cited, may be due to technical differences in the systems such as a "suboptimal" ratio of enzyme to substrate in this study. The conditions for 11hydroxylation by acetone-dried ox adrenal mitochondria has been well described by Grant (33).

A limited degree of activity has been detected in postmortem material in these studies. Grant, Symington and Duguid (34) failed to find any, however, when employing conditions similar to ours. The use of postmortem material is not generally advisable, and was employed here for purposes of comparison. Under these circumstances, it is especially noteworthy that specimen Sh retained its ability to hydroxylate at C-11 (but not C-21). Tissue which has been frozen at once and properly stored is satisfactory when suitable cofactors are added $(10,11,35)$.

\section{SUMMARY}

The conversion of 17-hydroxyprogesterone to 11-desoxycortisol and cortisol by normal human adrenal tissue, with a greater yield of the former, has been described. The adrenal glands from two normotensive cases of the adrenogenital syndrome, due to congential adrenocortical hyperplasia, failed to effect hydroxylation of 17-hydroxyprogesterone at either carbon 11 or 21 . On the other hand, when 11-desoxycortisol was employed as a substrate, both the normal and abnormal adrenal glands were able to bring about a significant conversion to cortisol. A relatively large quantity of 17-hydroxyprogesterone (but no 21-desoxycortisol) was found in the two glands from patients with the adrenogenital syndrome, without prior addition of substrate or incubation. These data directly confirm earlier indirect work pointing to a lack of "21-hydroxylase," and the presence of "11-hydroxylase" in the adrenal glands of patients with the normotensive form of this disorder. The results also suggest that 21-hydroxylation occurs prior to 11-hydroxylation in man. It has also been indicated that postmortem adrenal tissue, obtained within 24 hours of death, is capable of accomplishing the described conversions to a limited degree.

\section{ACKNOWLEDGMENT}

The infrared spectroscopy was possible through the kindness of Doctors S. Solomon and S. Lieberman. The collaboration of Doctors C. E. Koop, Walter R. Eberlein and Bernard Wagner is gratefully acknowledged. Steroids employed in these experiments were gifts from the Chemistry Division, Upjohn Company, Kalamazoo, Mich.

\section{REFERENCES}

1. Eberlein, W. R., and Bongiovanni, A. M. Congenital adrenal hyperplasia: An inborn error of metabolism. Helv. paediat. Acta 1956, 11, 105.

2. Bongiovanni, A. M., and Eberlein, W. R. Defective steroidal biogenesis in congenital adrenal hyperplasia. Pediatrics 1958, 21, 661.

3. Bartter, F. C., Albright, F., Forbes, A. P., Leaf, A., Dempsey, E., and Carroll, E. The effects of adrenocorticotropic hormone and cortisone in the adrenogenital syndrome associated with congenital adrenal hyperplasia: An attempt to explain and correct its disordered pattern. J. clin. Invest. 1951, 30, 237.

4. Eberlein, W. R., and Bongiovanni, A. M. Partial characterization of urinary adrenocortical steroids 
in adrenal hyperplasia. J. clin. Invest. 1955, 34, 1337.

5. Ely, R. S., Kelley, V. C., and Raile, R. B. Studies of 17-hydroxycorticosteroids in children. I. Peripheral blood levels in health and disease. $\mathrm{J}$. Pediat. 1953, 42, 38.

6. Bongiovanni, A. M., and Clayton, G. W., Jr. A simplified method for the routine determination of pregnanediol and pregnanetriol in urine. Bull. Johns Hopk. Hosp. 1954, 94, 180.

7. Fukushima, D. K., and Gallagher, T. F. Steroid isolation studies in congenital adrenal hyperplasia. J. biol. Chem. 1957, 229, 85.

8. Dorfman, R. I. Adrenal steroid metabolism in The Hormones, G. Pincus and K. V. Thimann, Eds. New York, Academic Press, 1955, vol. III, p. 589.

9. Tomkins, G. M., Curran, J. F., and Michael, P. J. Adrenal 11-beta hydroxylation (abstract). Fed. Proc. 1958, 17, 323.

10. Hayano, M., and Dorfman, R. I. The enzymatic C-11 beta-hydroxylation of steroids. J. biol. Chem. 1953, 201, 175.

11. Hayano, M., and Dorfman, R. I. The action of adrenal homogenates on progesterone, 17-hydroxyprogesterone and 21-desoxycortisone. Arch. Biochem. 1952, 36, 237.

12. Allen, W. M. A simple method for analyzing complicated absorption curves, of use in the colorimetric determination of urinary steroids. J. clin. Endocr. 1950, 10, 71.

13. Porter, C. C., and Silber, R. H. A quantitative color reaction for cortisone and related 17, 21-dihydroxy20-ketosteroids. J. biol. Chem. 1950, 185, 201.

14. Eberlein, W. R., and Bongiovanni, A. M. New solvent systems for the resolution of corticosteroids by paper chromatography. Arch. Biochem. 1955, $59,90$.

15. Bongiovanni, A. M., Eberlein, W. R., and Thomas, P. Z. Use of an organic base in the Zimmermann reaction. J. clin. Endocr. 1957, 17, 331

16. Eberlein, W. R., and Bongiovanni, A. M. Plasma and urinary corticosteroids in the hypertensive form of congential adrenal hyperplasia. J. biol. Chem. 1956, 223, 85.

17. Wilson, H., and Fairbanks, R. Chromogenic values of 17-hydroxy corticosteroids in a modified PorterSilber reaction. Arch. Biochem. 1954, 53, 71.

18. Gornall, A. G., and Macdonald, M. P. Quantitative determination of the steroid hormones with 2,4 dinitrophenylhydrazine. J. biol. Chem. 1953, 201, 279.

19. Hechter, O., and Pincus, G. Genesis of the adrenocortical secretion. Physiol. Rev. 1954, 34, 459.

20. Neher, R. Aldosterone and other adrenocortical hormones in human adrenals in International Symposium on Aldosterone. Boston, Little, Brown \& Co., 1958, p. 11
21. Wilkins, L. The diagnosis of the adrenogenital syndrome and its treatment with cortisone. J. Pediat. $1952,41,860$

22. Hayano, M., Dorfman, R. I., and Prins, D. A. Metabolism of the steroid hormones. Conversion of desoxycorticosterone to glycogenic material in vitro. Proc. Soc. exp. Biol. (N. Y.) 1949, 72, 7 C0.

23. Savard, K., Green, A. A., and Lewis, L. A. Oxidation of 11-desoxycorticosteroids with adrenal tissue homogenates. Endocrinology 1950, 47, 418.

24. McGinty, D. A., Smith, G. N., Wilson, M. L., and Worrel, C. S. The biosynthesis of 17-hydroxycorticosterone from 11-desoxy-17-hydroxycorticosterone. Science 1950, 112, 506.

25. Sweat, M. L. Enzymatic synthesis of 17-hydroxycorticosterone. J. Amer. chem. Soc. 1951, 73, 4056.

26. Hayano, M., Dorfman, R. I., and Yamada, E. Y. The conversion of desoxycorticosterone to glycogenic material by adrenal homogenates. J. biol. Chem. 1951, 193, 175.

27. Haines, W. J. III. Aspects of steroid hormone chemistry and physiology. Studies on the biosynthesis of adrenal cortex hormones. Recent Prog. Hormone Res. 1952, 7, 255.

28. Dorfman, R. I., Hayano, M., Haynes, R., and Savard, $\mathrm{K}$. The in vitro synthesis of adrenal cortical steroids in Ciba Foundation Colloquia on Endocrinology. Boston, Little, Brown \& Co., 1953, vol. VII, p. 191.

29. Plager, J. E., and Samuels, L. T. The conversion of progesterone to 17-hydroxy-11-desoxycorticosterone by fractionated adrenal homogenates. J. biol. Chem. 1954, 211, 21.

30. Lombardo, M. E., Roitman, E., and Hudson, P. B. Conversion of progesterone, 17-hydroxyprogesterone and 11-desoxycortisol to cortisol by the human adrenal gland in vitro. $\mathrm{J}$. clin. Endocr. 1956, 16, 1283.

31. Berliner, M. L., Berliner, D. L., and Dougherty, T. F. Metabolism of progesterone by adrenal tissue from patients with Cushing's syndrome and mammary carcinoma. J. clin. Endocr. 1957, 18, 109.

32. Brownie, A. C., Grant, J. K., and Davidson, D. W. The in vitro enzymic hydroxylation of steroid hormones. Biochem. J. 1954, 58, 218.

33. Grant, J. K. The in vitro enzymatic hydroxylation of steroids. 4. The role of fumarate and triphosphopyridine nucleotide in the enzymatic $11 \beta$-hydroxylation of 11-desoxycorticosterone. Biochem. J. 1956, 64, 559.

34. Grant, J. K., Symington, T., and Duguid, W. P. Effect of adrenocorticotropic therapy on the $11 \beta$ hydroxylation of desoxycorticosterone by human adrenal homogenates. J. clin. Endocr. 1957, 17, 933.

35. Hayano, M. Personal communication. 